\title{
Linear and Nonlinear Wave-Current Interactions over Constant Water Depth
}

\author{
X. Feng
}

\begin{abstract}
Nowadays, the exploration of the ocean energy has become necessary and attracted more and more attention by the researchers all over the world due to the pollution and energy depletion issues caused by the consumption of fossil energies. As designing and hydrodynamic analysis of the energy converters in physical experimental tank are both time consuming and expensive, many researchers have developed numerical wave tanks to investigate the problem of wave and current interacting with the energy converters. In this paper, a numerical wave and current tank of the viscous fluid with constant depth is established based on the Reynolds-Averaged Navier-Stokes (RANS) equations with $k-\varepsilon$ turbulence closure scheme. The volume of fluid (VOF) method is applied to accurately capture the water free surface. The wave generation, wave absorption and current absorption are accomplished by using the analytic relaxation approach. Based on the numerical wave and current tank established here, the linear wave and nonlinear wave-current interactions are simulated and analyzed. The result shows that the wave generated by using the analytic relaxation approach has the feature of high quality and stability. The numerical results of the linear wave-current interaction are compared with the analytical solution based on the perturbation method, which shows that the numerical wave and current tank established here is accurate and valid. Finally, the influence of current velocity on the wave parameters and the variation of wave crests with wave slopes for linear and nonlinear wave- current interactions are also numerically investigated.
\end{abstract}

Index Terms - Wave-current interaction, analytic relaxation approach, numerical wave and current tank, user defined function.

\section{INTRODUCTION}

Ocean energy-one type of renewable, clean and green energy has attracted more and more attention due to its huge energy potential. However, there still exist many technical issues and barriers in the exploration of the ocean energy. In order to solve these issues, it is important to carry out the

Manuscript received May 28, 2017; revised August 4, 2017. This work was supported in part by the National Natural Science Foundation of China with Grant (Project nos.:51409032 and 51409031), the Fundamental Research Funds for the Central Universities (Project nos.: 3132017006 , 3132016314) (China).

The author is with the Marine Engineering Department, Dalian Maritime University, Dalian, China (e-mail: 530546168@163.com). hydrodynamic analysis of the wave energy converters in wave and current conditions, which is studied based on experimental and physical models in the past. The physical experimental analysis are very limited due to the aspects of high cost (for establishing the experimental wave tank and for flow measuring), inconvenience for maintenance and management, hardness for monitoring of some specific flow data leading to the ignorance of some important details. As the development of computer technology and numerical methods based on Computational Fluid Dynamics (CFD) has advanced, numerical simulation has become increasingly useful and powerful for showing the complicated flow fields encountered in developing the wave energy converters technology under water wave and current conditions. In recent years, there are many models used to describe the water surface waves, and the Navier-Stokes or Reynolds Averaged Navier-Stokes (RANS) equation model have been viable since there models employ the VOF (Volume of Fluids) method, which was developed to manage interfaces between multiple phases, i.e., water and air and works well for the establishment of the numerical wave tank[1]. The Navier-Stokes or RANS equations based model solves the complete momentum equation, resolving vertical components as well as nonlinear terms and viscous and turbulent stresses. The Navier-Stokes equations can accurately describe water wave problems, but the direct numerical simulation (DNS) of the Navier-Stokes equations which solves the entire spectrum of motions( ranging from large eddy motions to the smallest turbulence scale motions) as well as nonlinear terms, viscous and turbulent stresses, has low computational efficiency and always is restricted to the hardware limitations. ReynoldsAveraged Navier-Stokes (RANS) equations [2], [3], which was firstly applied in marine technology about 20years ago, are alternatives that hugely reduce computational load compared to that of DNS. The studies on establishing the numerical wave tank using the RANS equations have been increasing, among which three types of wave-makers have been developed: static boundary wave-maker, moving boundary wave-maker and internal wave-maker [4].

With the application of static boundary wave-maker, waves are generated at the inlet boundary given specified velocities and the water surface elevation derived from any wave theory formulation [5]. As pointed out by Pengzhi Lin [6], the static boundary wave-maker will produce obvious numerical dissipation due to the strong wave reflection.

With the application of moving boundary wave-maker, the motion of a realistic wave-maker (flap type wave maker or piston-type wave-maker) is numerically simulated by a moving boundary. For example, Finnegan and Goggins [7] simulated the linear water waves and wave-structure 
interactions using the flap-type wave-maker based on the Ansys CFX commercial software. In their models, the moving boundary wave-maker is based on unstructured grids where solid zones are not considered in the computational domain and the object surfaces are treated as boundary conditions. Due to the computational domain changes as the solid body moving toward or away from the fluid, a re-meshing is inevitable in each time step or after a large distortion of the generated grid, which should be avoided suggested by most researchers.

With the application of internal wave-maker, waves are generated by applying a source function within a designated region inside the computational zone not interacting with reflected waves and being able to be combined with various wave absorber methods. The source function method may be subdivided into analytical relaxation method, mass source function method and momentum source function method. The mass source function method and the momentum source function method were first proposed by Wei Ge based on Boussinesq-type equations and were developed based on Navier-Stokes equations or RANS equations [8]. The mass source function method based on the Navier-Stokes equations model was first proposed by Lin and Liu by using the finite difference method (FDM) on a staggered grid system. The VOF scheme was adopted to track the free surface. The proposed numerical wave tank was applied to generate various types of wave, and the rules of thumb for designing the source region have become a general principle [9], [10]. Nevertheless, a lot of numerical tests are needed for the mass source function designing for generating a particular wave. The momentum source function method proposed firstly by Junwoo Choi [11] is more convenient than the mass source function method because the width of the momentum source domain is the only parameter to be specified, whereas the mass source function method has three (i.e., height, width, and location of the mass source domain). Mayer et al. [12] proposed the analytical relaxation method for generating and absorbing waves simultaneously in the relaxation domain. The analytical relaxation method is usually linked to the numerical sponge layer method, and thus the relaxation zone should be enlarged.

These methods have solved a lot of pure wave problems. However, wave and current often coexist in the practical engineering cases, and interaction between wave and a current flow plays an important role in accurate predictions of wave loads and responses of wave energy converters in waves and currents. The coexistence of current can significantly alter the magnitude and frequency of wave loading and body responses The mechanics of wave-current interaction has been extensively investigated in the past. Among these, Markus et al. [13] also adopted a CFD solver to simulate wave-current interaction, but they focused on the evaluation of flow field of a nonlinear wave in combination with a non-uniform current. J.-S. Zhang et al. [14] developed a numerical model for studying the wave propagation in the presence of a steady current flow based on the RANS equations, and investigated the effects of wave period and current velocity on regular wave-current induced water surface profile and velocity distribution. The application of the analytical relaxation methods based on the Navier-Stokes or RANS equations model for simulating the wave propagation on uniform currents over constant water depth have been reported in few studies.

In this study, a Reynolds-Averaged Naiver-Stokes (RANS) solver with finite-volume scheme, VOF method and $\mathrm{k}-\varepsilon$ turbulence model is developed to establish the numerical wave and current tank for simulating the wave propagation on uniform currents over constant water depth. A steady current is achieved by uniform velocity inflow boundary and pressure outflow boundary, while the desired waves are generated by incorporating source function derived based on the analytical relaxation method into momentum equations, which are achieved by using the User Defined Function (UDF) model in the software FLUENT. To avoid the reflection of incident wave and interference of inflow current, the analytic relaxation wave absorption approach is employed. The numerical wave and current tank established here is validated by comparing the numerical results with the analytical solutions, and then is further applied to investigate the effects of current velocity on the wave parameters and the wave slopes on wave crests for linear and nonlinear wave-current interactions.

\section{MAthematicAl ModeL}

\section{A. Governing Equations}

For the incompressible fluid motion due to wave-current interaction with the free surface, the governing equations are the RANS equations and mass conservation equations [15]:

$$
\begin{gathered}
\frac{\partial\left(u_{i}\right)}{\partial x_{i}}=0 \\
\frac{\partial\left(\rho u_{i}\right)}{\partial t}+\frac{\partial\left(\rho u_{i} u_{j}\right)}{\partial x_{j}}=\frac{\partial}{\partial x_{j}}\left(\mu\left(\frac{\partial u_{i}}{\partial x_{j}}+\frac{\partial u_{j}}{\partial x_{i}}\right)\right)-\frac{\partial p}{\partial x_{i}} \\
+\frac{\partial}{\partial x_{j}}\left(-\rho \overline{u_{i}^{\prime} u_{j}^{\prime}}\right)+\rho g_{i}+S_{i}
\end{gathered}
$$

in which $t$ is time and $\rho$ is the density, $x_{i}$ denotes the Cartesian coordinates, $u_{i}$ is an ensemble mean velocity component, $p$ is the fluid pressure, $\mu$ is the dynamic viscosity, and $g$ is the acceleration due to gravity. $S_{m}$ is the additional mass source term, $S_{i}$ is the additional momentum source term. $-\rho \overline{u_{i}^{\prime} u_{j}^{\prime}}$ is the Reynolds stress term, which is modeled by the sophisticated $\mathrm{k}-\varepsilon$ turbulence model. By applying eddy-viscosity assumption, the Reynolds stress term can be calculated by:

$$
-\rho \overline{u_{i}^{\prime} u_{j}^{\prime}}=\mu_{t}\left\lfloor\frac{\partial u_{i}}{\partial x_{j}}+\frac{\partial u_{j}}{\partial x_{i}}\right\rfloor-\frac{2}{3} \rho \delta_{i j} k
$$

in which $\mu_{t}$ is the turbulent viscosity, $\mu_{t}=\rho C_{\mu} k^{2} / \varepsilon$, in which $C_{\mu}=0.09, k$ is the turbulence kinetic energy and $\delta_{i j}$ is the Kronecker delta. The turbulence kinetic $k$ and turbulence dissipation rate $\varepsilon$ in equation $(2,3)$ should be satisfied with the following transport equations [16]: 


$$
\begin{aligned}
\frac{\partial(\rho k)}{\partial t} & +\rho k\left(\frac{\partial u_{i}}{\partial x_{i}}+\frac{\partial u_{j}}{\partial x_{j}}\right)=\left(\mu+\frac{\mu_{t}}{\sigma_{k}}\right)\left(\frac{\partial^{2} k}{\partial x_{i}^{2}}+\frac{\partial^{2} k}{\partial x_{j}^{2}}\right)+G_{k}-\rho \varepsilon \\
& \frac{\partial(\rho \varepsilon)}{\partial t}+\rho \varepsilon\left(\frac{\partial u_{i}}{\partial x_{i}}+\frac{\partial u_{j}}{\partial x_{j}}\right)=\left(\mu+\frac{\mu_{t}}{\sigma_{\varepsilon}}\right)\left(\frac{\partial^{2} \varepsilon}{\partial x_{i}^{2}}+\frac{\partial^{2} \varepsilon}{\partial x_{j}^{2}}\right) \\
& +\frac{C_{1 \varepsilon} \varepsilon}{k} G_{k}-C_{2 \varepsilon} \rho \frac{\varepsilon^{2}}{k}
\end{aligned}
$$

in which $G_{k}$ represents the generation of turbulence kinetic energy due to the mean velocity gradients, $\sigma_{k}$ and $\sigma_{\varepsilon}$ are the turbulent Prandtl numbers for $k$ and $\varepsilon$, respectively. $C_{1 \varepsilon}$ and $C_{2 \varepsilon}$ are constants. In the model calculations, the constants $C_{1 \varepsilon}, C_{2 \varepsilon}, \sigma_{k}$ and $\sigma_{\varepsilon}$ have the following default values: $C_{1 \varepsilon}=1.44, C_{2 \varepsilon}=1.92, \sigma_{k}=1, \sigma_{\varepsilon}=1.3$. These default values have been determined from experiments with air and water for fundamental shear flows including homogeneous shear flows and decaying isotropic grid turbulence. They have been found to work fairly well for a wide range of wall-bounded and free shear flows [17].

The VOF method is used to track the free water surface. The main idea of VOF method is to define a function $\alpha$ to represent the fractional volume of water fluid:

$$
\frac{\partial \alpha}{\partial t}+\frac{\partial\left(u_{i} \alpha\right)}{\partial x_{i}}=0
$$

$\alpha=1$ indicates that the cell is full of water, while $\alpha=0$ corresponds to a cell fully of occupied by air. Cells with value of $0<\alpha<1$ contain a water free surface.

\section{B. Analytic Relaxation Approach}

The analytic relaxation wave generation approach proposed by Mayer and Madson was used here for wave generation and wave absorption. As one of the internal wave generation method, it avoids the simultaneous specification of wave and current at inflow boundary and eliminating the effects of wave reflection. For the wave absorber, it works well without changing the water depth and the current velocity at outflow boundary. The mechanism of the analytical relaxation method can be described as following: Within the relaxation domain, the velocity will be updated at every time step by the added source. Within the wave generating region, the relaxation algorithm for velocity can be renewed as:

$$
u_{M}=C u_{J}+(1-C)\left(u_{L}+U\right), v_{M}=C v_{J}+(1-C) v_{L}
$$

in which the subscript $M$ stands for the renewed value of the physical quantity in the specified zone, the subscript $J$ stands for the computed value at previous time step, the subscript $L$ denotes the incoming wave, $U$ denotes the current velocity, $C=C(x)$ is the relaxation function, $C_{x \min }=0, C_{x \max }=1$, given by $C=\sin \left(x^{*} \pi / 2\right)$.

Within the wave absorbing region, the relaxation algorithm for velocity can be renewed as:

$$
u_{M}=C u_{J}+(1-C) U, v_{M}=C v_{J}
$$

in which $C_{x \min }=1, C_{x \max }=0$.

The additive momentum source term is determined by the
Euler equations ignoring the water viscosity. The difference forms of momentum equations with and without the additive source can be written as follow:

$$
\begin{gathered}
\rho \frac{u_{M}^{n+1}-u_{J}^{n}}{\Delta t}+\rho u_{J} \frac{\partial u_{J}}{\partial x}+\rho v_{J} \frac{\partial u_{J}}{\partial z}=-\frac{\partial p_{J}}{\partial x}+S_{x} \\
\rho \frac{v_{M}^{n+1}-v_{J}^{n}}{\Delta t}+\rho u_{J} \frac{\partial v_{J}}{\partial x}+\rho v_{J} \frac{\partial v_{J}}{\partial z}=-\frac{\partial p_{J}}{\partial z}+S_{z}-\rho g \\
\rho \frac{u_{M}^{n+1}-u_{M}^{n}}{\Delta t}+\rho u_{M} \frac{\partial u_{M}}{\partial x}+\rho v_{M} \frac{\partial u_{M}}{\partial z}=-\frac{\partial p_{M}}{\partial x} \\
\rho \frac{v_{M}^{n+1}-v_{M}^{n}}{\Delta t}+\rho u_{M} \frac{\partial v_{M}}{\partial x}+\rho v_{M} \frac{\partial v_{M}}{\partial z}=-\frac{\partial p_{M}}{\partial z}-\rho g
\end{gathered}
$$

Therefore, the additive momentum source term can be derived from equations (9) to (12). For zone I, the additive momentum source term can be expressed as:

$$
\begin{aligned}
& S_{x}=\frac{\rho}{\Delta t}(C-1)\left(u_{J}^{n}-u_{L}^{n}-U\right) \\
& +\rho(1-C)\left(\begin{array}{l}
\frac{1}{\rho} \frac{\partial p_{J}}{\partial x}+\frac{\partial\left(u_{L}+U\right)}{\partial t} \\
+\left(u_{L}+U\right) \frac{\partial\left(u_{L}+U\right)}{\partial x}+v_{L} \frac{\partial v_{L}}{\partial z}
\end{array}\right)
\end{aligned}
$$

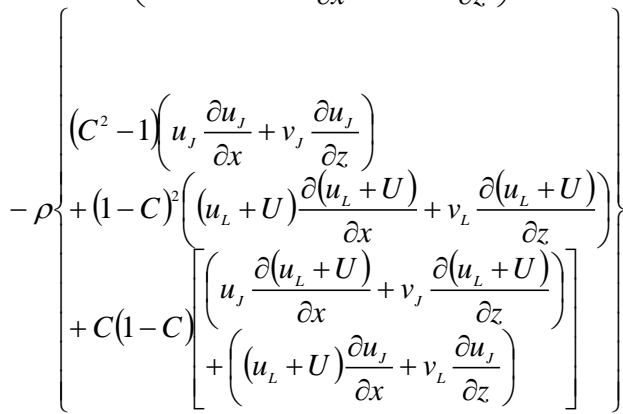

$$
\begin{aligned}
& S_{z}=\frac{\rho}{\Delta t}(C-1)\left(v_{J}^{n}-v_{L}^{n}\right) \\
& +\rho(1-C)\left(\begin{array}{l}
\frac{1}{\rho} \frac{\partial p_{J}}{\partial z}+\frac{\partial v_{L}}{\partial t}+ \\
\left(u_{L}+U\right) \frac{\partial v_{L}}{\partial x}+v_{L} \frac{\partial v_{L}}{\partial z}+g
\end{array}\right)
\end{aligned}
$$

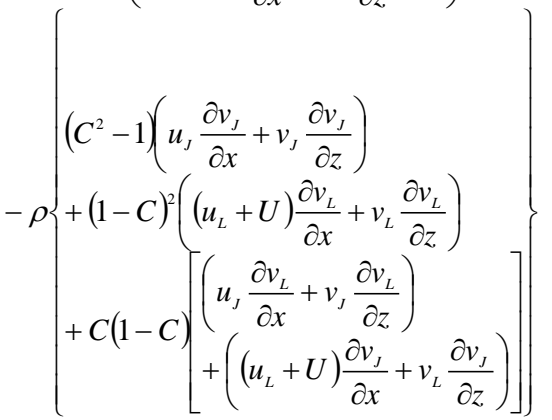

For the wave absorption zone II, the additive momentum source term can be expressed as:

$$
\begin{aligned}
& S_{x}=(1-C)\left(\frac{\partial p_{J}}{\partial x}-\rho \frac{u_{J}-U}{\Delta t}\right)+\rho\left(1-C^{2}\right)\left\lfloor u_{J} \frac{\partial u_{J}}{\partial x}+v_{J} \frac{\partial u_{J}}{\partial z}\right\rfloor \\
& -\rho^{*} C^{*}(1-C) * u^{*} u_{J} \\
& S_{z}=(1-C)\left(\frac{\partial p_{J}}{\partial z}-\frac{\rho v_{J}}{\Delta t}\right)+\rho\left(1-C^{2}\right)\left[u_{J} \frac{\partial v_{J}}{\partial x}+v_{J} \frac{\partial v_{J}}{\partial z}\right\rfloor \\
& -\rho^{*} C^{*}(1-C) * U^{*} v_{J}
\end{aligned}
$$


More details can be found in Wang [18].

\section{NUMERICAL METHOD}

The model sketch of the numerical wave and current tank of the viscous fluid is shown in Fig. 1. EF is the steady water surface, the tank is $200 m$ long, $8 m$ deep, and the steady water depth is $6.2 \mathrm{~m}$. The computational zones are discrete by the structured grids using GAMBIT. The grid size $\Delta x$ in the $\mathrm{x}$-direction should be in the range of $L / 20 \sim L / 50$ ( $L$ is the wave length), and the grid size $\Delta z$ in the $z$-direction should be in the range of $H / 5 \sim H / 10$ ( $H$ is the wave height). $A B$ is the wave and current inlet boundary. $C D$ is the symmetric outlet boundary, Zone I is the wave generating zone and Zone II is the wave absorbing zone.
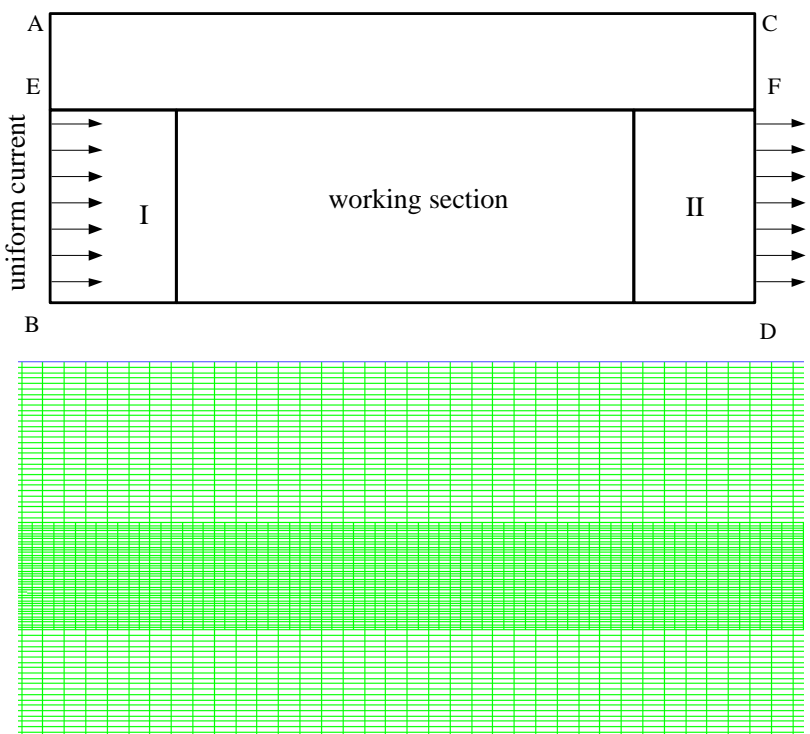

Fig. 1. Sketch map of computational case and part grid refinement.

In FLUENT software, finite volume method is employer for the discretion of governing equations on the basis o staggered grids. Pressure, turbulent kinetic energy, turbulen dissipation rate and water volume fraction function ar arranged at the center point of grid, fluid velocity component are arranged at the center point of corresponding gric boundary. In order to calculate convection and diffusior fluxes through the control volume faces, the PLIC-VOI algorithm is used for the reconstruction of the interface, an PISO algorithm is adopted for the solution of discret equations. The first-order and second-order accuracies are fo the time and space, respectively. The convergence criterion $\mathrm{i}$ set to 0.001 for all simulations. When the computationa errors reach the convergence limits, pressure, turbulen kinetic energy, turbulent dissipation rate and water volum fraction function distributions are obtained. By using th macro of the UDF-DEFINE_SOURCE (mom_source, cell thread, $d s$, eqn) in software FLUENT, the wave-generatin: and wave-absorbing zones are arranged properly.

\section{RESULTS AND DISCUSSION}

\section{A. Validation of the Numerical Wave and Current Tank}

To validate the accuracy of the numerical wave and current flume established here, two cases (linear wave with and without following current) are addressed. In these two cases, the following parameters are chosen: input wave height $H=0.6 m$, wave period $T=4 s$, corresponding wave length $\lambda=23.3 m$, current velocity $U=0$ (case-a), $U=0.3 \mathrm{~m} / \mathrm{s}$ (case-b).

In case-a, the flow field of the entire computational domain is initialized as $u=0, v=0$, and the turbulence specification method is used to define the values of turbulent kinetic energy $\mathrm{k}$ and turbulent dissipation rate $\varepsilon$ in the domain. The pressure of the entire computational domain is initialized as hydrostatic pressure distribution.

Fig. 2 shows the comparisons of time series of wave elevations between present model results and analytical results located at $x=120 \mathrm{~m}$ in case-a(wave alone case). The analytical wave elevations are calculated according to the following formula:

$$
\eta(x, t)=\operatorname{Re}\left\{A e^{i(k x-\omega t)}\right\}=A \cos (k x-\omega t)
$$

in which $A$ is the wave amplitude, $\omega$ is the wave frequency.

As shown in Fig. 2, the highly stable and repeated wave elevations can be obtained based on the numerical wave and current flume established above, and the numerical results compare well to the analytical results. A small discrepancy can be found due to the viscosity of the water wave particles not considered in the analytical results.
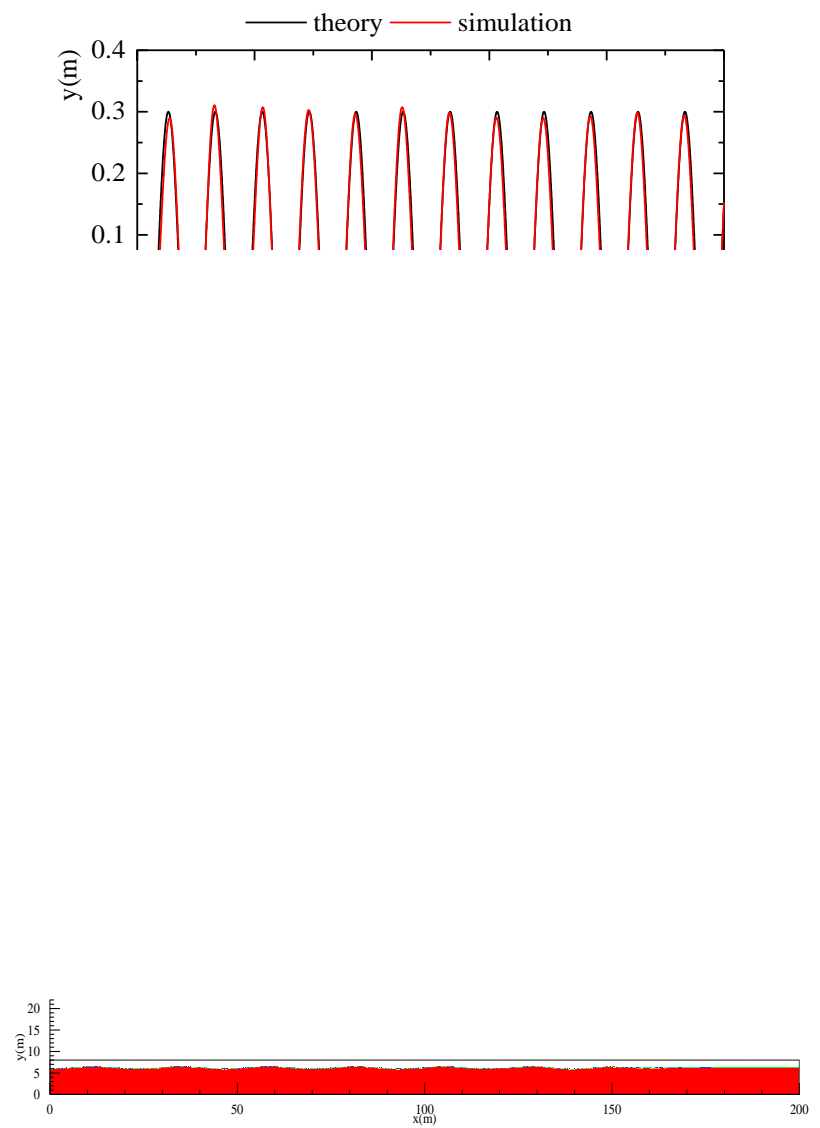

Fig. 3. Contour map of volume fraction in case-a.

Fig. 4 shows the path lines of water particles and iso-line map of $u$ and $v$. As shown in the figure, the orbits of the water wave particles are closed curves-ellipses, in comply with the description of the airy wave theory. 

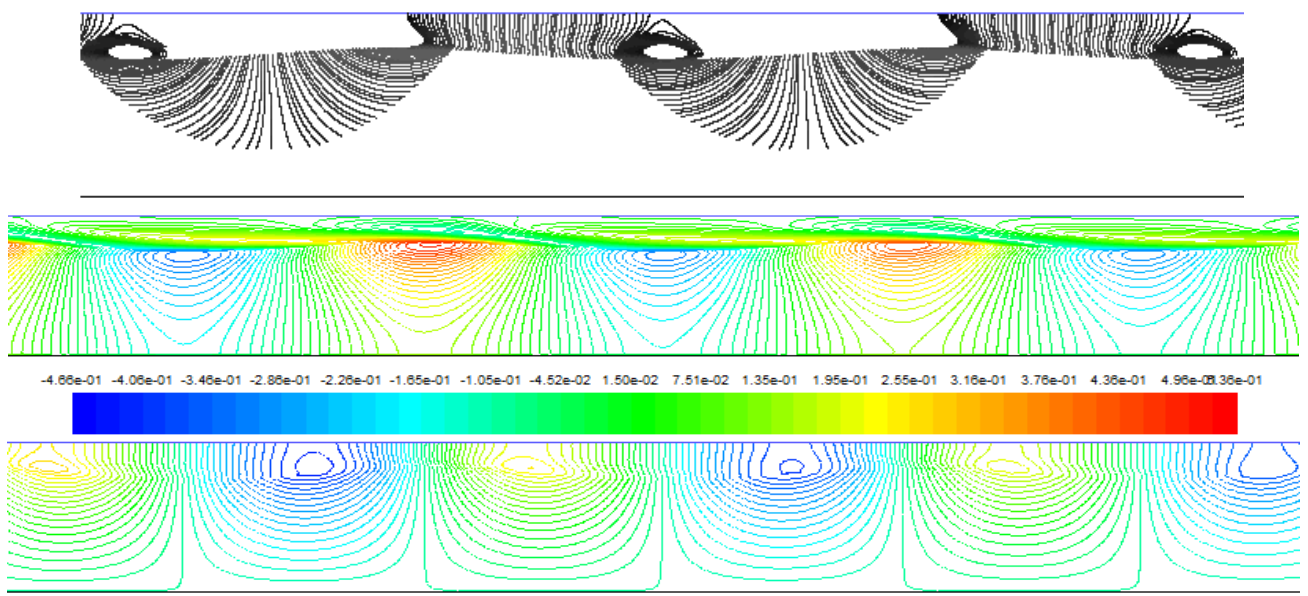

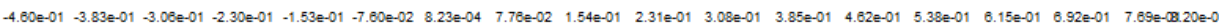

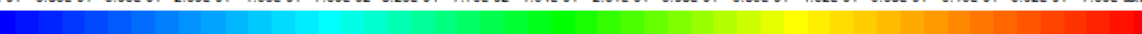

Fig. 4. Path lines of water particles and isoline map of $u$ and $v$.

In case-b, the flow field of the entire computational domain is initialized as $u=U, v=0, U$ is the current velocity, chosen to be $0.3 \mathrm{~m} / \mathrm{s}$.

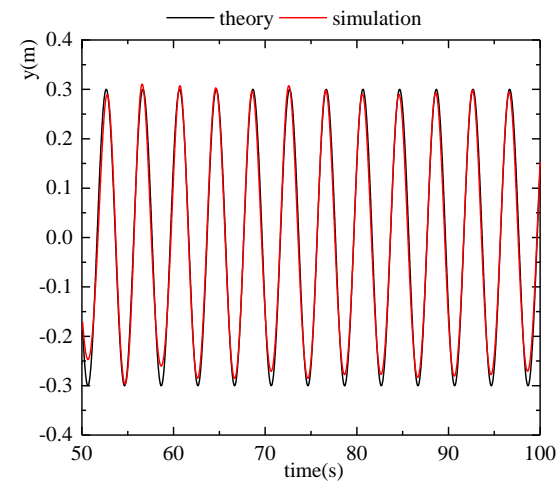

Fig. 5. Time series of wave elevations and comparisons of present model results and analytical results at $x=23.3 \mathrm{~m}$ in case-b.
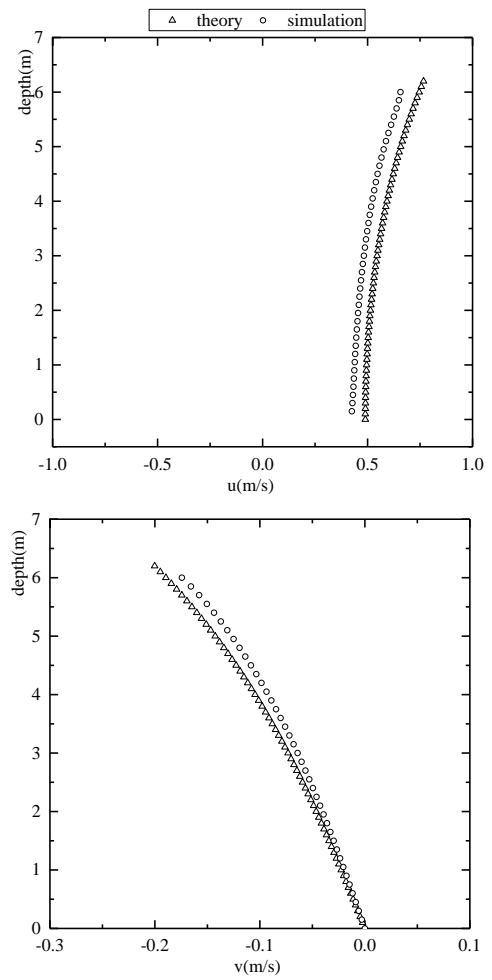

Fig. 6. Vertical distributions of $u$ and $v$ between numerical results and analytical results at time $t=6 \mathrm{~T}$ located at $x=23.3 \mathrm{~m}$.
Fig. 5 shows the comparisons of time series of wave elevations between present model results and analytical results located at $x=23.3 \mathrm{~m}$ in case-b-wave-current interaction case. The analytical wave elevations are calculated according to the following formula derived from the perturbation theory [19]:

$$
\eta=A \frac{\omega-k U}{\omega} \cos (k x-\omega t)
$$

Fig. 6 displays the comparison of simulated and analytical horizontal-velocity profiles and vertical-velocity profiles in wave-current interaction case. The numerical results comply well with the analytical results, so the validity of the numerical wave and current flume is further confirmed.

In general, the establishment of the numerical wave and current tank of the viscous fluid with constant depth was achieved successfully on the platform of software FLUENT based on the RANS equations for viscous, incompressible fluid and VOF method and analytic relaxation approach, and it works well for the simulations of the wave-current interactions.

\section{A. Verification of the Wave Absorption}

Fig. 7 shows the vertical distributions of $x$ - $(u)$ velocities at time $t=50$ s located at $x=190 \mathrm{~m}$, the numerical results compare well to the steady uniform current velocity. We can find that the analytic relaxation wave absorption zone works well for the eliminating effects of wave reflection in this numerical wave and current tank without changing the current velocity at the outflow boundary.

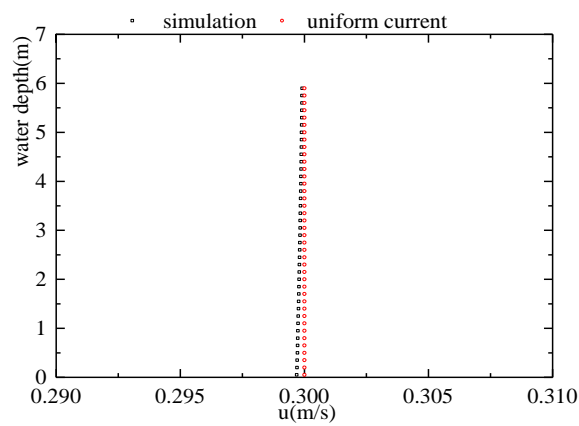

Fig. 7. Vertical distributions of $\mathrm{x}-(u)$ velocities between numerical results and steady uniform current velocity at time $t=50$ s located at $x=190 \mathrm{~m}$. 


\section{B. Nonlinear Wave-Current Interaction}

Then the NWCT was further applied for simulating the interactions between the solitary wave and uniform currents. The solitary wave propagation over constant water depth without current flows was firstly simulated. The case with input wave height $H=0.9 \mathrm{~m}$ is addressed. Fig. 8 shows the propagation direction of the generated solitary wave. Then the interactions between solitary wave on uniform currents over constant water depth were analyzed by setting the current velocity: $U= \pm 0.5 \mathrm{~m} / \mathrm{s}, \quad \pm 0.3 \mathrm{~m} / \mathrm{s}$. As shown in the Fig. 9, the induced wave heights of solitary wave are $0.96 \mathrm{~m}, 0.94 \mathrm{~m}$, $0.9 \mathrm{~m}, 0.88 \mathrm{~m}, 0.86 \mathrm{~m}$ for the cases with an opposing current ( $U=-0.5 \mathrm{~m} / \mathrm{s}, U=-0.3 \mathrm{~m} / \mathrm{s}$ ), no current and following current ( $U=0.3 \mathrm{~m} / \mathrm{s}, U=0.5 \mathrm{~m} / \mathrm{s}$ ), respectively. The change of the wave height induced by the opposing current is larger than that by the following current. Meanwhile, the traveling speed of the solitary wave is also changed by the ambient current flow according to the location of the wave crest found in Fig. 9.

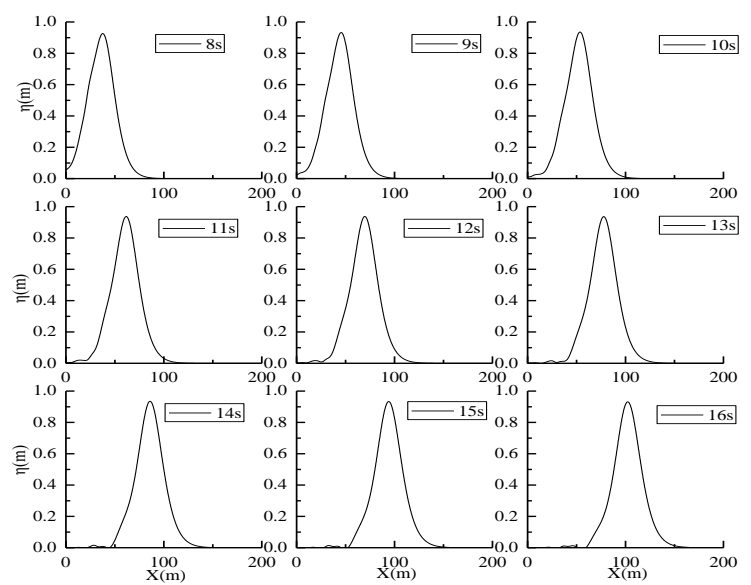

Fig. 8. Distribution of wave elevation along the symmetry of wave tank.

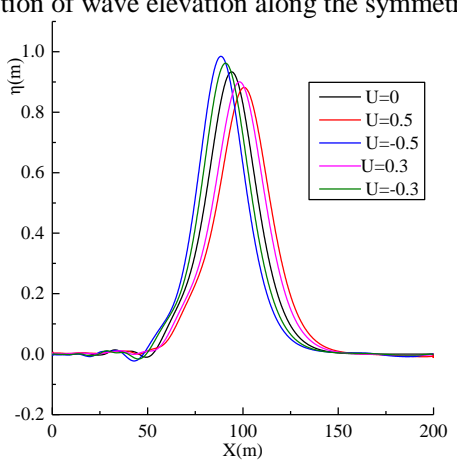

Fig. 9. Distribution of wave elevation along the symmetry of wave tank at time $T=15 \mathrm{~s}$ for different wave and current conditions.

\section{Effects of Current Velocity and Wave Slope}

More quantitative analysis is carried out to investigate the influence of current velocity on wave parameters. The input current velocities are chosen $U=0,0.15,0.2,0.23,0.3$ and 0.5 $(\mathrm{m} / \mathrm{s})$ respectively, and the wave is chosen as described above. Fig. 10 shows the rate of change of wave amplitude and wave length against current velocity for fixed waves, and the vertical axis is non-dimensionalized by dividing the wave parameters of the pure wave conditions. It can be observed that the wave length is increased and the wave amplitude is reduced by coplanar currents.

The numerical wave and current flume is next applied to investigate the variation of wave surface against current velocity for waves with different wave slopes. Five waves with different wave slopes are used to investigate how following current affect the wave elevations. The input values of these simulations are summarized in the Table I.

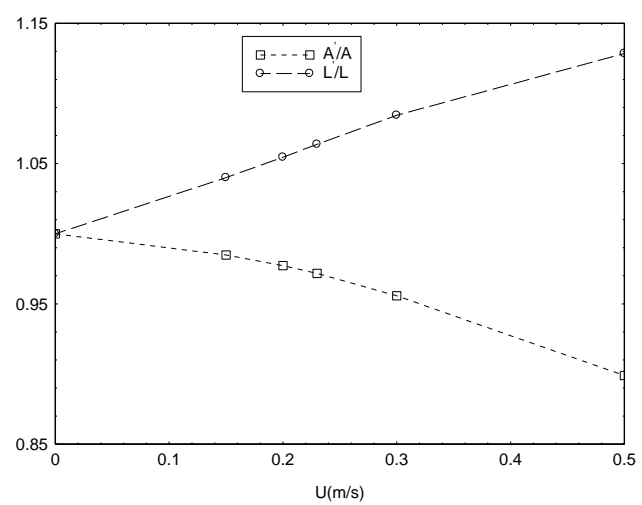

Fig. 10. Change of wave length and amplitude against current speed, L' (A')and L(A) represent wave length(wave amplitude) with and without current effect respectively.

TABLE I: INPUT PARAMETERS OF TWELVE CASES

\begin{tabular}{|c|c|c|c|c|c|}
\hline$A(\mathrm{~m})$ & $k A$ & $H / h$ & $U(\mathrm{~m} / \mathrm{s})$ & $T(\mathrm{sec})$ & $h(\mathrm{~m})$ \\
\hline 0.5 & 0.0788 & 0.16 & $\begin{array}{c}0 \\
0.3\end{array}$ & 5.8 & 6.2 \\
\hline 0.7 & 0.102 & 0.226 & $\begin{array}{c}0 \\
0.3\end{array}$ & 6.2 & 6.2 \\
\hline 0.3 & 0.0576 & 0.097 & $\begin{array}{c}0 \\
0.3\end{array}$ & 5 & 6.2 \\
\hline 0.5 & 0.0685 & 0.16 & $\begin{array}{c}0 \\
0.3\end{array}$ & 6.5 & 6.2 \\
\hline 0.5 & 0.085 & 0.16 & $\begin{array}{c}0 \\
0.3\end{array}$ & 5.5 & 6.2 \\
\hline
\end{tabular}

Fig.11 shows the variation of wave crests with wave slopes at $x=50 \mathrm{~m}$ under wave alone condition and wave-current interaction condition (linear and nonlinear), and the vertical axis is non-dimensionalized by dividing the wave parameters of the pure wave conditions. From this figure, the comparisons between linear and nonlinear computation for wave alone and wave-current interaction cases can be found. The nonlinear effect will increase as the wave slope increases for wave alone condition, but wave-current interaction will decrease the nonlinear effect.

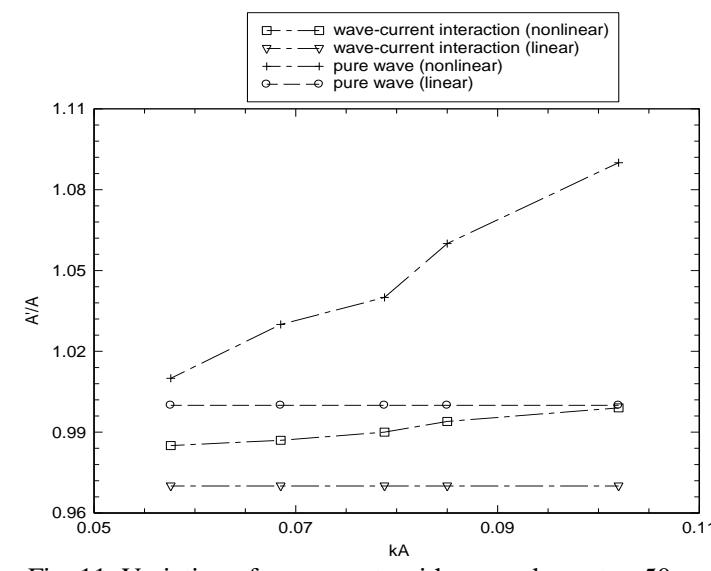

Fig. 11. Variation of wave crests with wave slope at $x=50 \mathrm{~m}$.

\section{CONCLUSION}

An effective and accurate approach- analytic relaxation 
approach in this study is developed for the simulation of wave-current interaction. Based on the analytic relaxation approach and RANS equations for viscous, incompressible fluid, a 2D numerical wave and current flume of the viscous fluid with constant water depth is established, utilizing the software FLUENT and its function of secondary development, and the wave interactions with steady uniform currents are studied. The performance of this numerical wave and current flume of the viscous fluid is verified by comparisons between the computations using the present model and the analytical results solved by the perturbation method. The good agreements between numerical simulation results and analytical results confirm that the established numerical wave and current flume is suitable for predicting the wave interactions with the steady uniform currents.

Based on the numerical wave and current flume, the linear and nonlinear wave-current interactions are analyzed, and the following can be drawn. When waves travel with a following current, the wave amplitudes decrease slightly and the wave length increase significantly. A larger current velocity results in a larger reduction in wave amplitude and larger increase in wave length. The propagation of a nonlinear wave can be largely affected by ambient current flow. The following current will reduce the nonlinearity of the wave-current interactions.

\section{REFERENCES}

[1] L. Shen and E.-S. Chan, "Numerical simulation of fluid- structure interaction using a combined volume of fluid and immersed boundary method," Ocean Engineering, vol. 35, pp. 939-952, 2008.

[2] P. Higuera, J. L. Lara, and I. J. Losada, "Simulating coastal engineering processes with OpenFOAM," Coast. Eng., vol. 71, pp. 119- 134, 2013

[3] S. C. Hsiao and T. C. Lin, "Tsunami-like solitary waves impinging and overtopping an impermeable seawall: Experiment and RANS modeling," Coast. Eng., vol. 57, pp. 1-18, 2010.

[4] P. Higuera, I. J. Losada, and J. L. Lara, "Three-dimensional numerical wave generation with moving boundaries," Coast. Eng., vol. 101, pp. 35- 47, 2015.

[5] P. Higuera, J. L. Lara, and I. J. Losada, "Realistic wave generation and active wave absorption for navier-stokes models: Application to OpenFOAM," Coast. Eng., vol. 71, pp. 102-118, 2013.
[6] P. Z. Lin and P. L.-F. Liu, "Internal wave-maker for Navier-Stokes equation models," Journal of Waterway, Port, Coastal, and Ocean Engineering, vol. 125, pp. 207-215, 1999.

[7] W. Finnegan and J. Goggins, "Numerical simulation of linear water waves and wave-structure interaction," Ocean Eng., vol. 43, pp. 23- 31, 2012.

[8] G. Wei, J. T. Kirby, and A. Sinha, "Generation of waves in Boussinesq models using a source function method," Coast. Eng., vol. 36, pp. 271-299, 1999.

[9] H. L. Wu, S. C. Hsiao, W. Y. Hsu, R. Y. Yang, and H. H. Hwung, "Dynamic response of density stratified fluid in a submarine rectangular trench," J. Hydro-environ. Res., vol. 9, pp. 61-80, 2015.

[10] H. L. Wu, S. C. Hsiao, and T. C. Lin, "Evolution of a two-layer fluid for solitary waves propagating over a submarine trench," Ocean Eng., vol. 110, pp. 36- 50, 2015.

[11] J. Choi and S. B. Yoon, "Numerical simulations using momentum source wave-maker applied to RANS equations model," Coast. Eng., vol. 56, pp. 1043-1060, 2009.

[12] S. Mayer, A. Garapon, and L. S. Soerensen, "A fractional step method for unsteady free surface flow with applications to non-linear wave dynamics," Int. J. Numer. Methods Fluids, vol. 28, pp. 293- 315, 1998

[13] D. Markus, M. Hojjat, R. Wuechner, and K. U. Bletzinger, "A CFD approach to modeling wave-current interaction," Int.J.Offshore Polar Eng., vol. 23, pp. 29-32, 2013

[14] J.-S. Zhang, Y. Zhang, D.-S. Jeng, P. L.-F. Liu, and C. Zhang, "Numerical simulation of wave-current interaction using a RANS solver," Ocean Eng., vol. 75, pp. 157-164, 2014.

[15] P. D. Hieu, P. N. Vinh, D. Van Toan, and N. T. Son, "Study of wave-wind interaction at a seawall using a numerical wave channel," Applied Mathematical Modelling, vol. 38, pp. 5149-5159, 2014.

[16] X. F. Liang and J. M. Yang, "A numerical study on local characteristics of predetermined irregular wave trains," Ocean Eng., vol. 38, pp. 651-657, 2011.

[17] FLUENT Inc., User's Guide, 2010.

[18] B. Wang, "Mathematical modelling of surf zone based on the high order boussinesq equations," Ph.D thesis, Shanghai Jiao Tong University, Shanghai, China, 2010.

[19] Z. L. Zou, P. C. Hu, and K. C. Fang, "Boussinesq-type equations for wave- current interaction,” Wave Motion, vol. 50, pp. 655-675, 2013.

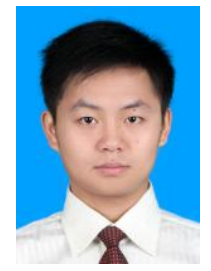

X. Feng was born in 1985 in Linchuan, China. $\mathrm{He}$ received his doctor degree in Dalian Maritime University. After his ten years studying in marine engineering, he worked as a teacher at DMU. Now, he is conducting research about CFD analysis of the floating structure in ocean waves and currents. 